\title{
Relação entre intensidade da corrida, percepção de esforço e estados de humor em corredores recreacionais
}

\section{Relationship between race intensity, rate of perceived exertion and mood states in recreational runners}

\author{
João Henrique Gomes ${ }^{1}$ \\ Renata Rebello Mendes ${ }^{2}$ \\ Roberta Luksevicius Rica ${ }^{3}$ \\ Rafael M. Pitta ${ }^{4}$ \\ Rafael Ambrósio Battazza ${ }^{5}$ \\ Gerson Leite ${ }^{6}$ \\ Alex Souto Maior ${ }^{7}$ \\ Danilo Sales Bocalini ${ }^{8}$
}

\section{Endereço científico}

Universidade Federal de Sergipe (UFS), São Cristóvão/SE, Brasil - Av. Marechal Rondon,

s / n - Jd. Rosa Elze, São Cristóvão - SE, 49100-000

\section{Endereço para correspondência}

Danilo Sales Bocalini

Instituição: Universidade Federal do Espirito Santo. Centro de Educação Física e Desporto Av. Fernando Ferrari, 514, Campus Universitário, Goiabeiras. 29075810 - Vitoria, ES [Brasil].bocaliniht@hotmail.com

Programa de Pós-Graduação em Ciencias da Saúde da Universidade Federal de Sergipe (UFS). Aracaju, SE - Brasil. profjhgomes@gmail.com

2 (iD) Departamento de Nutrição da Universidade Federal de Sergipe (UFS). Aracaju, SE - Brasil.

mendes@hotmail.com

Departamento de Educação Física da Universidade Estacio de Sá (UES). Vitoria, ES - Brasil.

robertarica@hotmail.com

iD Departamento de Medicina Preventiva do Hospital Albert Ainstein, São Paulo (HAA). São Paulo, SP - Brasil. rafael.pitta@einstein.br

5 iD Programa de Pós Graduação em Ciencias da Reabilitação da Universidade Nove de Julho (UNINOVE). São Paulo, SP - Brasil. rafaelbattazza@hotmail.com

6 (iD Departamento de Educação da Universidade Nove de Julho (UNINOVE). São Paulo, SP - Brasil.

gersonslt@gmail.com

(iD) Centro Universitário Augusto Motta (UNISUAM). Rio de Janeiro, RJ - Brasil.

alex.bioengenharia@gmail.con

8 (iD) Centro de Educação Fisica e Desporto da Universidade Federal do Espirito Santo (UFES). Vitoria, ES - Brasil. bocaliniht@hotmail.com

\begin{abstract}
Resumo
Introdução: a avaliação da percepção de esforço (PSE) e estados de humor (EH) em diferentes intensidades de esforço, pode auxiliar o planejamento do programa de treinamento minimizando o risco de overtraining e lesões osteomioarticulares. Objetivo: comparar as respostas de PSE e estados de humor em individuos submetidos a duas intensidades distintas de corrida de $5 \mathrm{~km}$.

Métodos: corredores de rua recreacionais foram submetidos a um teste de $5 \mathrm{Km}$. Todos os individuos foram submetidos a dois testes de $5 \mathrm{~km}$ randomizados com diferentes intensidades de esforço do teste de $5 \mathrm{~km}$. Foram avaliados o tempo total, a veocidade media, a percepçaõ de esforço, a carga interna e os parámetros vigor e fadiga.

Resultados: o protocolo de corrida C5K85 apresentou redução significativa $(\mathrm{p}<0,001)$ das variáveis velocidade média de corrida, PSE e CIT quando comparado ao protocolo C5K95. A variável fadiga apresentou aumento significativo $(\mathrm{p}<0,001)$ pós-esforço no protocolo C5K95, sem mudanças significativas no protocolo C5K85 ( $\mathrm{p}>0,05)$.

Conclusão: a percepção subjetiva de esforço, a carga interna de treino e a subescala fadiga do questionário BRUMS podem ser utilizados como ferramentas para controle e monitoramento da intensidade de treinamento em corredores de $5 \mathrm{~km}$.
\end{abstract}

Descritores: Corrida. Afeto. Desempenho físico funcional. Exercício físico. Teste de esforço.

\begin{abstract}
Introduction: The evaluation of the perception of effort (PE) and mood states of humor (MS) in different intensities of effort, can aid the planning of the training program, minimizing the risk of overtraining and osteomioarticular lesions.

Objective: Evaluated of PE and MS responses in healthy individuals submitted to two distinct $5 \mathrm{~km}$ run intensities.

Methods: Recreational street runners from the Race Club of the Federal University of Sergipe were submitted to a $5 \mathrm{Km}$ test. After the test, all the subjects were submitted to two $5 \mathrm{~km}$ randomized tests with different intensities the test speed of $5 \mathrm{~km}$. Total time, mean velocity, PE, internal load, and force and fatigue parameters were evaluated. Results: The $85 \%$ running protocol presented a significant reduction $(\mathrm{p}<0.001)$ in the variables mean running velocity, PE and ITC when compared to the $95 \%$ protocol. The fatigue variable presented a significant increase $(\mathrm{p}<0.001)$ post-effort on $95 \%$ protocol, without significant changes in the $85 \%$ protocol $(\mathrm{p}>0.05)$,

Conclusion: The PE, the internal training load and the fatigue subscale of the BRUMS questionnaire can be used as tools to control and monitor training intensity in $5 \mathrm{~km}$ runners.
\end{abstract}

Keyword: Running. Affect. Physical functional performace. Exercises. Exercise Test.

\section{Cite como}

Gomes JH, Mendes RR, Rica RL, Pitta RM, Battazza RA, Leite G et al. Relação entre intensidade da corrida, percepção de esforço e estados de humor em corredores recreacionais. Conscientiae Saúde 2019 jul./set.; 18(3):301-311. https://doi.org/10.5585/ConsSaude.v18n3 11179. 


\section{Introdução}

No meio esportivo, o controle da carga interna de treinamento é um aspecto importante para monitorar as adaptações fisiológicas relacionadas aos efeitos das cargas externas, consequentemente, minimizar o risco de overtraining e lesão ${ }^{1}$. Desta forma, os ajustes nas cargas de treinamentos, a partir de ferramentas metodológicas que contribuem com o feedback do atleta, tornam-se fundamentais para prescrição e monitoramento do treinamento. Por exemplo, durante a corrida de rua a intensidade pode ser entendida principalmente pela velocidade, tipos de pisos, aclives, declives, direção do vento, humidade relativa do ar, ou seja, fatores externos que afetam diretamente a carga interna do individuo ${ }^{2,3}$. Assim, é imprescindível considerar a carga interna para melhor compreensão do estímulo aplicado ${ }^{4-6}$. Atualmente, existem diversas formas de monitorar a intensidade enquanto o indivíduo está correndo, seja por monitoramento da frequência cardíaca ou intensidade da corrida. Ambas formas dependem de equipamentos específicos (monitores cardíacos e GPS) que possuem alto custo e difícil acesso a maioria da populaçãó ${ }^{2}$.

Portanto, observa-se a necessidade de se controlar a carga interna de corredores através de métodos de fácil aplicação ${ }^{7}$. Nessa perspectiva, a percepção subjetiva de esforço (PSE) e os questionários subjetivos estado de humor têm sido sugeridos para controlar a carga interna em diversas modalidades esportivas, seja na prática recreacional ou competitiva ${ }^{8,9}$.

Estudos com atletas de basquetebol ${ }^{10,11}$, voleibol $^{12}$, judocas ${ }^{13}$, entre outros têm apresentado altas correlações entre a PSE e marcadores fisiológicos de fadiga, dano muscular e estresse oxidativo. Especificamente na corrida, Kluitenberg et al. ${ }^{14}$, examinaram as associações entre a PSE, variáveis de treinamento (intensidade, volume e frequência) e lesões em corredores iniciantes. Os pesquisadores concluiram que correr em intensidades mais altas apresentava relação direta com o aumento da PSE, consequentemente, maior risco de lesão em corredores iniciantes. Por outro lado, são limitadas ou escassas as investigações que procuraram relacionar a PSE e o estado de humor com a intensidade dos treinos de corrida. O conhecimento das respostas de PSE e estados de humor em diferentes intensidades de esforço, poderia contribuir no acompanhamento da carga de treinamento e auxiliar o planejamento adequado para os estímulos futuros, além de minimizar o risco de overtraining e lesões osteomioarticulares $^{1}$. Sendo assim, o objetivo do presente estudo foi investigar e comparar as respostas de PSE e estados de humor em individuos submetidos a duas intensidades distintas de corrida de $5 \mathrm{~km}$. 


\section{Métodos}

Amostra

Os critérios de inclusão foram: 1) ter idade entre 18 e 45 anos; 2) ter participado de uma prova de $5 \mathrm{~km}$ nos últimos três meses com tempo máximo de 35 minutos para cumprir a prova; 3) estar treinando corrida regularmente com frequência mínima de três vezes por semana com no minimo 150 minutos semanais; 4) não possuir histórico de lesão nos últimos três meses. Os critérios de exclusão foram: 1) apresentar qualquer dor ou lesão musculoesquelética que pudesse comprometer a realização das corridas durante a avaliação e os protocolos; 2) Ausência nos dias da aplicação dos protocolos; 3) Apresentar sinais de infecções respiratórias ou outra disordem da saúde que pudesse impedir a realização dos treinos de corrida.

Antes de participar da pesquisa os voluntários preencheram um questionarios com informações sobre estado de saude, receberam informações sobre os objetivos e procedimentos metodológicos, alem de aceitarem a participação desde que aptos a participarem do estudo, leram e assinaram o termo de consentimento livre e esclarecido. O presente estudo respeitou as normas da Declaração de Helsinki e assinaram Termo de Consentimento Livre e Esclarecido para a participação, que foi aprovado pelo Comitê de Ética em Pesquisa da Universidade Federal de Sergipe, processo $\mathrm{n}^{\circ} 2.535 .181 / 2018$. Desta forma, 29 corredores demonstraram interesse no estudo porem apos aplicação dos criterios de exclusão dois individuos apresntaram lesão musculoesquelética e dois individuos apresentaram e infecções respiratórias, totalizando 25 corredoderes. Contudo apenas 14 individuos participaram do estudo, sendo 11 excluidos para não comparecerem na sessão ou por participar em apenas uma sessão. A tabela 1 apresenta os valores médios \pm desvio padrão das principais características dos participantes do estudo e do tempo total do TC5KM. 
Tabela 1 - Características antropométricas e desempenho dos corredores

\begin{tabular}{lc}
\hline \multicolumn{2}{c}{ Variáveis } \\
\hline Idade (anos) & $31,4 \pm 8,3$ \\
Estatura $(\mathrm{cm})$ & $175,6 \pm 7,5$ \\
Massa corporal $(\mathrm{kg})$ & $79,7 \pm 14,1$ \\
Índice de Massa Corporal $\left(\mathrm{kg} / \mathrm{m}^{2}\right)$ & $25,8 \pm 2,5$ \\
Massa gorda $(\mathrm{kg})$ & $14,8 \pm 6,2$ \\
Massa gorda $(\mathrm{kg})$ & $14,8 \pm 6,2$ \\
Percentual de gordura corporal $(\%)$ & $18,6 \pm 5,9$ \\
Teste de simulação de corrida 5km (min:s) & $28: 20 \pm 3: 21$ \\
\hline Valores expressos em media \pm DP. & \\
Fonte: Os autores.
\end{tabular}

\section{Delineamento experimental}

No presente estudo os voluntários realizaram três visitas ao Departamento de Educação Física da Universidade Federal de Sergipe (DEF-UFS), em ocasiões separadas para avaliações e execução dos dois protocolos de treino. Na primeira visita ocorreram as avaliações antropométricas e a realização da prova simulada de corrida de $5 \mathrm{~km}$. As duas visitas subsequentes objetivaram avaliar a PSE e o estado de humor dos sujeitos através de dois distintos protocolos de treinamento de corrida. A fim de garantir que os sujeitos estivessem em condições similares de recuperação na avaliação e execução dos protocolos de treinamento, foi padronizado intervalo de 72 horas sem a execução de qualquer exercício físico. Todas as avaliações e protocolos de treinamento foram realizadas no horário habitual de treinamento dos corredores (17h) e conduzidas pelos mesmos pesquisadores na pista oficial de atletismo da UFS. Foram necessários 7 dias para avaliações e aplicabilidade dos dois protocolos de treinamento na amostragem total.

Teste de simulação de corrida $5 \mathrm{~km}$ (TC5KM)

Para avaliação da performance dos corredores de rua recreacionais foi utilizado o TC5KM, adaptado de Frainer, Oliveira, e Pazin ${ }^{15}$, na pista oficial de atletismo do DEF-UFS. Antes da execução da simulação de prova de $5 \mathrm{~km}$ em esforço máximo, foi realizado 
aquecimento padronizado com trote por 5 minutos em intensidade moderada de acordo com a escala de Borg. Nenhum tipo de alongamento ou exercício educativos de corrida foram permitidos. Durante o TC5KM, os participantes foram encorajados a correr o mais rápido possível a cada volta realizada na pista. Afim de manter um ritmo de corrida o mais constante possível, foi permitido aos participantes utilizarem relógios com tecnología de GPS, cronômetro ou aplicativo de celular específico para corrida. O tempo foi mensurado utilziado cronometro padrão

\section{Protocolo de treinamento}

Foram adotados dois protocolos de treinamento de corrida com o mesmo volume (5 $\mathrm{km}$ ), porém, com intensidades. Os distintos protocolos forma caracterizados como: C5K95 (corrida a 95\% da velocidade média do TC5KM) e C5K85 (corrida a 85\% da velocidade média do TC5KM). A ordem de aplicação dos protocolos de foi aleatorizada (sorteio) afim de minimizar possíveis vieses. Não foi encontrado diferenças nos parámetros ambientais (poluição e umidade do ar, temperatura, pressão, força do vento e visibilidade) entre os días em que foram realizados os testes (dados não mostrados)

\section{Carga interna da sessão de treino}

A carga interna da sessão de treinamento (CIT) foi determinada utilizando a escala de Borg CR-10 ${ }^{16}$, de acordo com o método da PSE, proposto por Foster ${ }^{17}$. Após 30 minutos do final de cada sessão de treinamento de corrida, o sujeito indicava, individualmente, por meio da escala CR10, o grau do esforço físico percebido. A fim de calcular a CIT, o escore relatado pelos sujeitos foi multiplicado pelo tempo total da sessão de treino em minutos ${ }^{18}$.

\section{Escala de Humor de Brunel (BRUMS)}

A Escala de Humor de Brunel contém 24 indicadores de humor organizados em seis subescalas ou dimensões: raiva (estado de hostilidade em relação aos outros), confusão (estado de tontura e instabilidade nas emoções), depressão (estado emocional de desânimo, tristeza e infelicidade), fadiga (estado de cansaço e baixa enegia), tensão (estado da tensão musculoesquelética e preocupação) e vigor (estado de energia e disposição física). Os avaliados 
respondem, de acordo com uma escala tipo Likert de 5 pontos (sendo $0=$ nada, $1=$ um pouco, $2=$ moderamente, $3=$ bastante, e $4=$ extremamente). A pontuação total de cada subescala pode variar de 0 a 16.

Para orientar as respostas, foi utilizada a pergunta "Como você se sente agora?". Como o intuito foi avaliar a percepção dos indivíduos em diferentes intensidade de esforço, utilizamos para as análises somente as sub-escalas Vigor e Fadiga ${ }^{19,20}$. O BRUMS levou cerca de um a dois minutos para ser respondido, sendo realizado antes e 30 min pós-esforço para ambos os protocolos. Ressalta-se que a fim de evitar a influência de um corredor sobre outro, bem como garantir a correta interpretação do instrumento, o pesquisador avaliou individualmente os corredores, de forma que não havia comunicação entre eles até que todos fossem avaliados.

\section{Análises estatística}

A estatística descritiva em valores médios e desvio padrão foi utilizada. $\mathrm{O}$ teste de Shapiro-wilk foi aplicado para testar a normalidade dos dados. Utilizou-se o Teste T de Student e ANOVA duas vías conforme necessario. A correlação linear de Pearson foi utilizada na identificação de possíveis correlações entre a PSE com as subescalas vigor e fadiga, bem como da CIT com as subescalas vigor e fadiga. Os dados foram analisados usando o SPSS versão 21.0. A significância adotada foi de $p<0,05$

\section{Resultados}

A media do teste de TC5KM foi de 28,20 $\pm 3,21$ minutos, a intensidade realizada nas duas intervencões representou $96,4 \%$ e $83,7 \%$, para a CK95 e CK85, respectivamente em relação ao TC5KM.

A tabela 2 apresenta os valores médios \pm desvio padrão do desempenho, PSEE CIT nos protocolos 95\% e 85\%. O protocolo de corrida C5K85 apresentou redução significativa ( $\mathrm{p}<$ 0,001) das variáveis velocidade média de corrida, PSE e CIT quando comparado ao protocolo C5K95. Por outro lado, o protocolo C5K85 apresentou aumento significativo do tempo total (p $<0,001)$. 
Tabela 2- Variáveis de desempenho, PSE e CIT na C5K95 e C5K85

\begin{tabular}{lccc}
\hline \multicolumn{1}{c}{ Variáveis } & C5K95 & C5K85 & Significância \\
\hline Tempo total (min) & $29,04 \pm 4,00$ & $33,26 \pm 4,36$ & $\mathrm{p}=0,001$ \\
Velocidade média (m/s) & $2,92 \pm 0,41$ & $2,54 \pm 0,36$ & $\mathrm{p}=0,001$ \\
PSE (UA) & $8,9 \pm 1,2$ & $5,1 \pm 2,0$ & $\mathrm{p}=0,001$ \\
CIT (UA) & $261,8 \pm 52,8$ & $177,0 \pm 87,9$ & $\mathrm{p}=0,001$
\end{tabular}

Valores expressos em media \pm DP. PSE = perceção de esforço. CIT = carga interna da sessão de treinamento (tempo total da sessão de treino em minutos x PSE). UA = unidade arbitrária Fonte: Os autores.

A tabela 3 apresenta os valores médios \pm desvio padrão dos estados de humor vigor e fadiga antes e após a execução das corridas nas intensidade de $95 \%$ e $85 \%$. A variável fadiga apresentou aumento significativo ( $\mathrm{p}<0,001)$ pós-esforço no protocolo C5K95, sem mudanças significativas no protocolo C5K85 (p>0,05). Não foi evidenciado

Tabela 3- Estados de humor vigor e fadiga antes e após C5K95 e C5K85

\begin{tabular}{llllll}
\hline & \multicolumn{3}{c}{ C5K95 } & \multicolumn{3}{c}{ C5K85 } \\
Variables & Antes & Depois & Antes & Depois \\
\hline Vigor & $8,4 \pm$ & $8,8 \pm \pm$ & $7,1 \pm$ & $8,9 \pm$ \\
(UA) & 3,3 & 3,0 & 3,0 & 3,3 & \\
Fadiga & $2,6 \pm$ & $9,1 \pm \pm$ & $1,8 \pm$ & $2,9 \pm$ \\
(UA) & 2,4 & $3,7^{*}$ & 1,4 & 2,3 & \\
\hline
\end{tabular}

Valores expressos em media \pm DP. UA: unidade arbitrária.

$* \mathrm{p}<0,01$ vs antes. $\neq \mathrm{p}<0,01$ vs CK 85 .

Fonte: Os autores.

Conforme descrito na tabela 4 foi encontrado correlação significante entre a PSE e fadiga, tanto na C5K95 quanto na CK85, bem como entre a PSE e CIT. As demais correlações foram não significantes $(\mathrm{p}>0,05)$ em ambas intervenções. 
Tabela 4- Correlação entre os parámetros de estados de humor e indicadores de carga de treinamento nos testes de C5K95 e C5K85

\begin{tabular}{lcccc}
\hline & PSE & CIT & Vigor & Fadiga \\
\hline CK95\% & - & & & \\
PSE & $0,75^{*}$ & $0,75^{*}$ & $-0,09$ & $0,64^{*}$ \\
CIT & $-0,09$ & 0,07 & 0,07 & 0,51 \\
Vigor pós & $0,64^{*}$ & 0,51 & - & 0,27 \\
Fadiga pós & & & 0,27 & - \\
CK85\% & - & $0,97^{*}$ & $-0,20$ & $0,54^{*}$ \\
PSE & $0,97^{*}$ & - & $-0,04$ & 0,52 \\
CIT & $-0,20$ & 0,04 & - & 0,24 \\
Vigor pós & $0,54^{*}$ & 0,52 & 0,24 & - \\
&
\end{tabular}

\section{Discussão}

O principal achado do nosso estudo foi identificar que a percepção subjetiva de esforço, a carga interna de treino e a subescala fadiga do questionário BRUMS podem ser utilizados como ferramentas para controle e monitoramento da intensidade de treinamento em corredores de $5 \mathrm{~km}$.

No mesmo sentido Wallace et al. ${ }^{22}$ demonstram a aplicabilidade da PSE, sugerindo que o incremento desta, na sessão de treinamento para uma carga externa pre-determinada, serviria como indicador de fadiga excessiva ou de diminuição da capacidade de trabalho do atleta. Por outro lado, a redução da PSE, na sessão de treinamento para a mesma carga predeterminada, indicaria adaptação ao treinamento. Além disso, estudos recentes demonstram que a utilização da PSE e BRUMS, na sessão do treinamento, incluem a possibilidade dos treinadores avaliarem e compararem, individualmente ou coletivamente, o nível de estresse relacionado aos diversos componentes na mesma ${ }^{22,23}$. Nesse sentido estudos recentes demonstraram a correlação positiva entre a PSE e marcadores fisiológicos de esforço, tais como a frequência cardíaca e a concentração sanguínea de Lactato em corredores de diferentes distâncias ${ }^{24}$.

Adicionalmente, estudos sugerem que a manipulação das variáveis subjetivas serviriam 
como instrumento de orientação, para os treinadores, na distribuição/organização das cargas de treinamento em diferentes etapas de uma periodização, tais como os períodos de intensificação, restauração e polimento, além de evitar o desenvolvimento do sobretreinamento e lesões, devido a correleção entre a fadiga, mensurada pela PSE e marcadores fisiológicos, hormonais e do sistema imune ${ }^{5}$.

Logo, tento em vista que o processo de monitoramento das cargas de treinamento é imprescindível na otimização do desempenho esportivo e além do baixo custo e fácil manipulação, a utilização dessas ferramentas tornam-se importantes no acompanhamento da sessão e periodização do treinamento.

Entretanto, a presente investigação apresenta limitações, tais como: a) ausência de monitoramento de marcadores fisiológicos, da alimentação, do uso de suplementos alimentares, do sono e de outras variáveis psicológicas (estresse, ansiedade, motivação, autoconfiança e autoestima); b) o número reduzido de participantes na pesquisa, que não permite extrapolar os resultados para outras populações. Contudo, embora o tamanho amostral reduzido, investigações na área do esporte tendem a ser mais complexas. $\mathrm{O}$ conjunto de limitações apresentados deve servir de base para a continuidade de investigações acerca deste assunto, considerando o monitoramento da carga de treinamento por meio da PSE e estados de humor com outras variáveis de desempenho.

\section{Conclusão}

Conclui-se que a percepção subjetiva de esforço, a carga interna de treino e a subescala fadiga do questionário BRUMS podem ser utilizados como ferramentas para controle e monitoramento da intensidade de treinamento em corredores de $5 \mathrm{~km}$. Intensidade de treino similar a intensidade da prova de corrida de $5 \mathrm{~km}$ apresenta percepção de esforço classificada como muito difícil e altos valores para a subescala fadiga, enquanto numa intensidade de $85 \%$ da intensidade da prova, esses valores reduzem. Já a subescala vigor não alterou quando intensidades diferentes de treinamento de corrida foram executadas. A PSE possuiu correlação com a subescala fadiga, por outro lado a CIT não apresentou correlação com essa mesma vairável. 


\section{Agradecimentos}

Os autores agradecem a Fundação de Amparo à Pesquisa do Espírito FAPES (590/19 - nº 84417625/2018) e a todos os corredores pela dedicação e participação. Agradecemos também a equipe de treinamento do Clube de corrida UFS por sua assistência e entusiasmo em relação ao projeto.

\section{Referências}

1. Halson SL. Monitoring training load to understand fatigue in athletes. Sports Med. 2014;44 Suppl 2:S139-47. doi:10.1007/s40279-014-0253-z

2. Mujika I. Quantification of Training and Competition Loads in Endurance Sports: Methods and Applications. Int J Sports Physiol Perform. 2017;12(Suppl 2):S29-S217. doi:10.1123/ijspp.2016-0403 3. Jensen K, Johansen L, Kärkkäinen OP. Economy in track runners and orienteers during path and terrain running. J Sports Sci. 1999;17(12):945-950. doi:10.1080/026404199365335

4. Ament W, Verkerke GJ. Exercise and fatigue. Sports Med. 2009. doi:10.2165/00007256200939050-00005

5. Nakamura FY, Moreira A, Aoki MS. Monitoramento da carga de treinamento: a percepção subjetiva do esforço da sessão é um método confiável? Rev da Educ Física/UEM. 2010. doi:10.4025/reveducfis.v21i1.6713

6. Foster C, Rodriguez-Marroyo JA, De Koning JJ. Monitoring training loads: The past, the present, and the future. Int J Sports Physiol Perform. 2017. doi:10.1123/IJSPP.2016-0388

7. Kelly VG, Coutts AJ. Planning and monitoring training loads during the competition phase in team sportd. Strength Cond J. 2007. doi:10.1519/00126548-200708000-00005

8. Rohlfs ICP de M, Carvalho T de, Rotta TM, Krebs RJ. Aplicação de instrumentos de avaliação de estados de humor na detecção da síndrome do excesso de treinamento. Rev Bras Med do Esporte. 2004;10(2):111-116. doi:10.1590/S1517-86922004000200005

9. Sties SW, Gonzáles AI, Schmitt Netto A, Wittkof PG, Lima DP, Carvalho T. Validação da escala de humor de brunel para programa de reabilitação cardiovascular. Rev Bras Med do Esporte.

2014;20(4):281-284. doi:10.1590/1517-86922014200401999

10. Arruda AFS, Moreira A, Nunes JA, Viveiros L, Junior DR, Aoki MS. Monitoramento do nível de estresse de atletas da seleção brasileira de basquetebol feminino durante a preparação para a copa américa 2009. Resvista Bras Med do Esporte. 2013;19(1):44-47.

doi:10.1590/S1517-86922013000100009

11. Gomes JH, Mendes RR, Polito LFT, Zanetti MC, Bocalini DS, Figueira Junior AJ. Mood State, Body Composition and Physical Performance of Young Basketball Players Through a Competition. J Phys Educ. 2018;29(1):1-12. doi:10.4025/jphyseduc.v29i1.2969

12. High A. Avaliação de Estados de Humor em Atletas de Tênis e Voleibol Jovens e Adultos de Alto 
Rendimento. Saúde Transform Soc. 2016;6(2):28-43.

13. Brandt R, Bevilacqua GG, Andrade A. Perceived Sleep Quality, Mood States, and Their Relationship with Performance among Brazilian Elite Athletes during a Competitive Period. J Strength Cond Res. 2017;31(4):1033-1039. doi:10.1519/JSC.0000000000001551

14. Kluitenberg B, van der Worp H, Huisstede BMA, et al. The NLstart2run study: Training-related factors associated with running-related injuries in novice runners. J Sci Med Sport. 2016. doi:10.1016/j.jsams.2015.09.006

15. Frainer DES, De Oliveira FR, Pazin J. Influência da maturação sexual, idade cronológica e índices de crescimento no limiar de lactato e no desempenho da corrida de 20 minutos. Rev Bras Med do Esporte. 2006. doi:10.1590/S1517-86922006000300006

16. Borg G. Escala de Borg Para Dor e o Esforço Percebido. 1ª edição. (Manole, ed.).; 2000.

17. Fisher G, Schwartz DD, Quindry J, et al. Lymphocyte enzymatic antioxidant responses to oxidative stress following high-intensity interval exercise. 2011:730-737. doi:10.1152/japplphysiol.00575.2010.

18. Foster C. Monitoring training in athletes with reference to overtraining syndrome. In: Medicine and Science in Sports and Exercise. ; 1998. doi:10.1097/00005768-199807000-00023

19. Rohlfs ICPDM, Carvalho T De, Rotta TM, Krebs RJ. Aplicação de instrumentos de avaliação de estados de humor na detecção da síndrome do excesso de treinamento. Rev Bras Med do Esporte. 2004;10(2):176-181. doi:10.1590/S1517-86922004000200005

20. Terry, P.C., Lane A. User Guide for the Brunel Mood Scale (BRUMS). (University of Southern Queensland, ed.).; 2010.

21. Dancey CP, Reidy J. Estatística Sem Matemática Para Psicologia.; 2006. doi:10.1017/CBO9781107415324.004

22. Wallace LK, Slattery KM, Coutts AJ. The ecological validity and application of the session-rpe method for quantifying training loads in swimming. J Strength Cond Res. 2009.

doi:10.1519/JSC.0b013e3181874512

23. Alexiou $\mathrm{H}$, Coutts AJ. A comparison of methods used for quantifying internal training load in women soccer players. Int J Sports Physiol Perform. 2008. doi:10.1123/ijspp.3.3.320

24. Piero DW di, Valverde-Esteve T, Redondo-Castán JC, Pablos-Abella C, Díaz-Pintado JVSA. Effects of work-interval duration and sport specificity on blood lactate concentration, heart rate and perceptual responses during high intensity interval training. PLoS One. 2018. doi:10.1371/journal.pone.0200690 